\title{
Effects of Tramadol on Liver and Renal Biochemistry and Histopathology in Dogs Undergoing Surgery under Pentobarbitone Anesthesia
}

\author{
${ }^{1}$ S. Buhari, ${ }^{1}$ H. Kalthum, ${ }^{2}$ Y.M. Goh, ${ }^{3}$ M.M. Noordin, ${ }^{1}$ H.C. Chen and ${ }^{4}$ S.H. Gan \\ ${ }^{1}$ Department of Veterinary Clinical Studies, ${ }^{2}$ Department of Veterinary Preclinical Studies, \\ ${ }^{3}$ Department of Veterinary Pathology and Microbiology, Faculty of Veterinary Medicine, \\ Universiti of Putra Malaysia, Serdang, Selangor, Malaysia \\ ${ }^{4}$ Human Genome Centre, School of Medical Sciences, Universiti of Sains Malaysia, \\ 16150 Kubang Kerian Kelantan, Malaysia
}

\begin{abstract}
Effect of single and repeated doses of tramadol on hepatic and renal functions and on pentobarbitone anesthesia was evaluated in dogs. Twenty five dogs were randomly distributed into five equal groups viz groups I and II received $3 \mathrm{mg} \mathrm{kg}^{-1}$ of tramadol by subcutaneous and intravenous injections, respectively during premedication alone. Groups III and IV received similar doses of tramadol during premedication which was repeated $2 \mathrm{~h}$ after the initial dose thorough subcutaneous and intravenous injections, respectively. Group $\mathrm{V}$ served as negative control. Blood samples $(2 \mathrm{~mL})$ were taken at 0,2 and $4 \mathrm{~h}$ after tramadol's administration while liver and kidney biopsies were taken before the surgery ended. Alanine Aminotransferase (ALT) and Aspartate Aminotransferase (AST) levels were significantly lowered in groups II-IV when compared to the group V at $4 \mathrm{~h}$. Significant increase in AST was observed in groups I, III and V at $2 \mathrm{~h}$ and at $4 \mathrm{~h}$ in groups I and V. No significant change in kidney profile. Histopathological changes in the liver and kidney biopsies are mainly congestion, edema and cellular infiltration which occurred less frequently in groups III and IV. The volume of pentobarbitone was significantly lowered in groups III and IV. It is concluded that repeated administrations of tramadol at $3 \mathrm{mg} \mathrm{kg}^{-1}$, IV or SC is safe at a frequency of $2 \mathrm{~h}$ interval during surgery without causing irreversible hepatic and renal damage and reduced the required dose of pentobarbitone needed to maintain anaesthesia in healthy dogs.
\end{abstract}

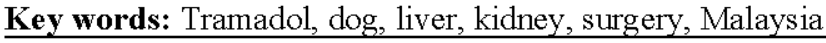

\section{INTRODUCTION}

There is an increasing concern in the control of acute and chronic pains in animals (Dehkordi et al., 2010; Wootton et al., 1988; Abu-Seida, 2012). Pain management is widely recognized as an essential component of clinical veterinary care in dogs and cats (Taylor, 2003). Currently, Nonsteroidal Anti-Inflammatory Drugs (NSAIDs) are the most widely used analgesics in dogs. Despite their easy availability and therapeutic advantages, NSAIDS' side effects limit their use (Kukanich and Papich, 2004). Furthermore, they are ineffective in managing severe surgical pains (Kukanich and Papich, 2004). The application of transdermal patches is not a useful alternative because of the higher cost and potential hazards (Riviere and Papich, 2001). Hence, opioids remain the analgesics of choice in the management of moderate to severe surgical pains (Atici et al., 2005; Kongara et al., 2010; Mercadante and Arcuri, 2004).
Tramadol is an analgesic with a dual mechanism of action. It binds to the $\mu_{1}$-opioid receptor (Guedes et al., 2005; Kukanich and Papich, 2004) as well as inhibits the monaminergic pathway, i.e., the Noradrenaline (NA) and serotonin (5HT) reuptake pathways (Kubota et al., 2008; McMillan et al., 2008). Due to this reason, tramadol is also coined as an atypical opioid and is only partially inhibited by the opioid receptor antagonist naloxone (McMillan et al., 2008).

The strategy for administering opioids and other groups of analgesics before surgery is referred as preemptive analgesia which is aimed at preventing central sensitization of nociception (Mastrocinque and Fantoni, 2003). Preemptive administration of tramadol is found to significantly reduce the requirements of isoflurane among human patients (Wordliczek et al., 2002) and sevoflurane in dogs (Seddighi et al., 2009). Tramadol is metabolized by an isoenzyme Cytochrome P-450 2D6 (CYP2D6) (Kukanich and Papich, 2004) to produce an

Corresponding Author: H. Kalthum, Department of Veterinary Clinical Studies, Faculty of Veterinary Medicine, Universiti of Putra Malaysia, Serdang, Selangor, Malaysia 
active metabolite O-desmethyltramadol (M1). This is mediated by the hepatic canine ortholog of CYP2D15 (Tasaki et al., 1998) before its rapid elimination by the kidneys (McMillan et al., 2008). Liver and kidneys are often predisposed to toxic injury due to their active role in drug metabolism and excretion. Both endogenous and exogenous opioids strongly influenced renal functions through various mechanism (Mercadante and Arcuri, 2004) leading to various degrees of injury in chronic administration (Atici et al., 2005). Due to the fact that tramadol has a rapid elimination half-life, more frequent dosage intervals are needed to potentiate effective pain control such as that caused by major surgeries (Kukanich and Papich, 2004; McMillan et al., 2008). However, a potential risk in increased peroxidation, hepatic and renal damage due to long use of opioids was reported in rats (Atici et al., 2005).

In recent years, interest in studying renal and hepatic function during anesthesia and surgery in small animals has increased greatly (Kongara et al., 2009). Despite the increased use of tramadol in the management of chronic and postoperative pain in animals, there is limited information on its effects on hepatic and renal functions following its repeated administration in prolonged surgical procedures in dogs. Many previous studies focused on physiological changes that occur during the surgery (Wunsch et al., 2010) which can be attributed to immediate problems associated with cardiopulmonary functions in most surgical procedures (Valtolina et al., 2009; Wunsch et al., 2010). Thus, to incorporate an appropriate analgesic protocol, it is essential that both laboratory and clinical investigations are taken into consideration. More importantly, the differential effect of tramadol in dogs differs from other species (Vettorato et al., 2010) and their increased sensitivity to NSAIDs (Lascelles, 1999). Therefore, the objective of this study was to investigate the effects of the repeated intraoperative administration of tramadol on hepatic and renal blood biochemistry and pathological changes in dogs and to determine its effect on pentobarbitone sodium's requirement for maintainence of anesthesia.

\section{MATERIALS AND METHODS}

Animals: Twenty five mongrel dogs of both sexes weighing between 10 and $19 \mathrm{~kg}(12.8 \pm 2.5)$ and ageing approximately between 1 and 4 years old $(2.3 \pm 0.8)$ were used in this study. They were allowed drinking water ad libitum until $2 \mathrm{~h}$ to the preanaesthetic medication while food was withheld $12 \mathrm{~h}$ before anaesthesia. The dogs were randomly assigned into five groups of equal number viz., groups that received either a single subcutaneous (group I) or single intravenous (group II) dose of tramadol (Unichem Laboratories Ltd. Ghaziabad India) at $3 \mathrm{mg} \mathrm{kg}^{-1}$ during premedication. The other two remaining groups were given either a repeated subcutaneous (group III) or a repeated intravenous premedication (group IV) of a dose of $3 \mathrm{mg} \mathrm{kg}^{-1}$ tramadol $2 \mathrm{~h}$ intra-operatively in addition to the premedication doses an hour before surgery. Group V without tramadol throughout the surgery acted as a negative control. Tramadol dosage was determined base on established data (Kukanich and Papich, 2004; McMillan et al., 2008). The dogs were physically examined by an experienced veterinary doctor before being included in the student surgery practice. This is a double blinded study where both the anaesthetist and surgeon were unaware about the treatment. Ethical approval for the study was granted by the University Animal Care Utility Committee of Universiti Putra Malaysia (UPM/FPV/PS/3.2.1.551/AUP-R86).

\section{Experimental protocol}

Anaesthesia and surgical protocols: Acepromazine maleate and atropine sulphate were administered subcutaneously to all dogs as a pre-anaesthetic agents at 0.1 and $0.04 \mathrm{mg} \mathrm{kg}^{-1}$, respectively. General anesthesia was induced with pentobarbitone sodium $\left(30 \mathrm{mg} \mathrm{kg}^{-1}\right)$ via the cephalic vein using $21 \mathrm{G}$ butterfly catheter within approximately $30 \mathrm{~min}$ from the time of pre-anaethesia. Pentobarbitone sodium was administered until jaw relaxation was sufficient to allow tracheal intubation. Anaesthesia was maintained using pentobarbitone sodium and the dogs were allowed to breath spontaneously in room air. Total volume of pentobarbitone sodium for induction and maintenance of anesthesia, body weight of dogs, duration of anesthesia and surgery were recorded.

Blood sampling and biopsy: Approximately, $2 \mathrm{~mL}$ of blood sample was collected from each dog into plain tube at baseline via a lateral saphenous vein. Subsequently, tramadol was administered followed by blood collection at two different time intervals ( 2 and $4 \mathrm{~h}$ ) via jugular venipuncture to avoid interruption with the surgery (exploratory laparatomy and ovariohysterectomy). All samples were collected into $3 \mathrm{~mL}$ plain tubes and the blood was allowed to clot at room temperature before being centrifuged immediately (1000 $\mathrm{g}$ for $10 \mathrm{~min})$ to separate the serum.

A biopsy sample from the hepatic margin was obtained by guillotine method (Theresa, 2007) at the end of surgery. Briefly, a loop of suture was placed at approximately $2 \mathrm{~cm}$ from the protruding margin of the liver lobe and the ligature was pulled tight to crush the hepatic parenchyma. The blood vessels and biliary ducts were properly ligated before a cut at an approximately $5 \mathrm{~mm}$ distal to the ligature was conducted. Using a No. 15 scapel blade, a wedge biopsy of the kidney was obtained 
by making two incisions into the renal parenchyma at an angle (approximately $30^{\circ}$ ) perpendicular to each other. The incision was closed in a mattress suture pattern using a 3-0 absorbable suture material. Finally, liver and kidney biopsies were immediately fixed in a $10 \%$ formalin for histopathology until further processing.

Serum chemistry analysis: Serum Alanine Aminotransferase (ALT), Aspartate Aminotransferase (AST), Lactate Dehydrogenase (LDH), concentration of Blood Urea Nitrogen (BUN) and serum creatinine levels were measured using an automatic biochemistry analyzer (Hitachi 902 automatic chemistry analyzer, United State) by kinetic UV assay, rate-blanked and compensated, urea/urea nitrogen and creatinine Jaffe method (Roche diagnostics $\mathrm{GmbH}$, Mannheim USA) according to the manufacturer's instructions (Atici et al., 2005).

Histopathologic examination: Following fixation, liver and kidney biopsies were automatically dehydrated in ascending grade of alcohol (ethanol), cleared in xylene (BDH Laboratory Pode, England) and embedded in paraffin wax (BDH Laboratory Pode, England). Samples were blocked with paraffin wax and then sectioned at $4 \mu \mathrm{m}$ using a microtome (Leica 2045, Germany). Following this the sections were mounted on glass slides and stained with hematoxylin (Sigma Eldrich, Germany) and eosin (Sigma Eldrich, Germany). The slides were dipped in xylene and the cover was slipped. Examination of the slides was carried out using a light microscope (Olympus BX51, Japan) which was coupled with an image analyzer (Computer Analysis LS Research).

Statistical analysis: Total volume of pentobarbitone sodium for induction and maintenance of anesthesia, body weight of dogs, duration of anesthesia and surgery were compared using one-way ANOVA. Serum chemistry parameters were compared across time and treatment groups using a two-way ANOVA test. All parameters were expressed as means $\pm \mathrm{SD}$ while histopathological changes were evaluated in terms of disease severity. Significant different means were examined using the Duncan multiple range test. All statistical analysis were conducted at 95\% confidence level using SPSS Version 16 (SPSS Inc. Chicago USA).

\section{RESULTS AND DISCUSSION}

Anesthesia and surgery record: Mean duration of surgery duration of anesthesia and body weight of the dogs were not significantly different within and between the groups. However, the mean volume of pentobarbitone sodium was significantly lowered in groups III and IV compare to groups I, II and V (Fig. 1a-d).
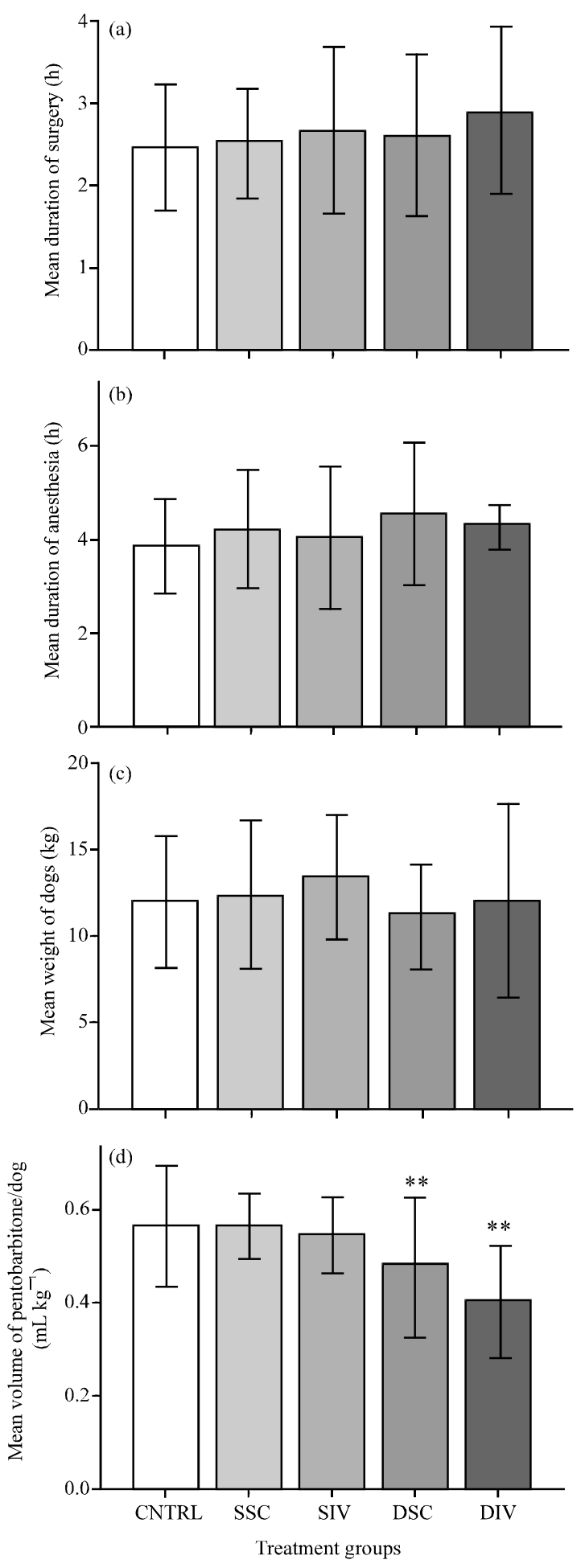

Fig. 1a-d: SSC Single Subcutaneous; SIV Single Intravenous; DSC repeated subcutaneous; DIV repeated intravenous; CNTRL Negative Control. **means with superscripts differ significantly at $\mathrm{p}<0.05$ between groups 
Table 1: Liver and kidney serum profiles of dogs at baseline and after tramadol administration in the different treatment groups

\begin{tabular}{|c|c|c|c|c|c|}
\hline Groups & $\begin{array}{l}\text { Time } \\
\text { (h) }\end{array}$ & $\begin{array}{c}\mathrm{ALT} \\
\left(\mathrm{U} \mathrm{L}^{-1}\right)\end{array}$ & $\begin{array}{c}\mathrm{AST} \\
\left(\mathrm{U} \mathrm{L}^{-1}\right)\end{array}$ & $\begin{array}{c}\text { BUN } \\
\left(\mathrm{mg} \mathrm{dL}^{-1}\right)\end{array}$ & $\begin{array}{l}\text { Creatinin } \\
\left.(\mu \mathrm{mol} \mathrm{L})^{-1}\right)\end{array}$ \\
\hline & 0 & $25.84 \pm 9.1600$ & $33.92 \pm 4.920$ & $7.30 \pm 1.02$ & $122.00 \pm 16.42$ \\
\hline & 2 & & & & \\
\hline & 4 & & & 0.00 & \\
\hline \multirow[t]{3}{*}{ IT } & 0 & $2:$ & 30.1 & 5.90 & \\
\hline & 2 & 2. & 0 & 5.76 & \\
\hline & 4 & 22.5 & & 5.64 & 61 \\
\hline & 0 & & & 5.02 & \\
\hline & 2 & & & & \\
\hline & 4 & & & 5.3 & \\
\hline \multirow[t]{2}{*}{ IV } & 0 & 23.88 & 28.04 & $5.14 \pm 2.33$ & $105.40 \pm 7.600$ \\
\hline & 2 & 23.66 & 25.70 & 2.13 & 92.0 \\
\hline & 0 & $24.06 \pm 10$ & & $4.94=$ & $102.60 \pm 18.52$ \\
\hline & 2 & & & & \\
\hline & 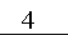 & $167.92 \pm 106.13^{\mathrm{a}}$ & $136.82 \pm 28.19^{a}$ & $5.50 \pm 0.75$ & $90.00 \pm 19$ \\
\hline
\end{tabular}

ALT $=$ Alanine Transaminase; AST Aspatate Transaminase; BUN $=$ Blood Urea Nitrogen; group I single subcutaneous; group II single intravenous; group III repeated subcutaneous; group IV repeated intravenous; group V negative control; 'means within columns with superscripts differ significantly at $\mathrm{p}<0.05$ between groups

Table 2: Histopathological changes in the liver among the various treated groups ( $n=5 /$ group)

\begin{tabular}{|c|c|c|c|c|c|}
\hline \multirow[b]{2}{*}{ Lesions } & \multicolumn{5}{|c|}{ Groups } \\
\hline & I & II & III & IV & $\mathrm{V}$ \\
\hline Cellular infiltration & 5 & 4 & 3 & 3 & 5 \\
\hline Edema & 3 & 4 & 2 & 2 & 3 \\
\hline Congestion & 2 & 3 & 1 & 1 & 5 \\
\hline Vacuolation & - & - & - & - & 4 \\
\hline Necrosis of hepatocytes & 1 & - & - & - & 3 \\
\hline
\end{tabular}

Group I single subcutaneous; group II single intravenous; group III repeated subcutaneous; group IV repeated intravenous; group V negative control. The numbers in the table indicates number of dogs affected in each group

Serum chemistry analysis: At $4 \mathrm{~h}$, serum ALT and AST were not significantly affected among the experimental groups (II-IV) when compared group V. In addition to a significant increase in serum AST level at $2 \mathrm{~h}$ of blood collection among groups I, III and V, a significant increase was also observed at $4 \mathrm{~h}$ among groups I and $\mathrm{V}$ (Table 1). However, no significant increase was found in BUN and serum creatinine levels in all of the groups throughout the sampling period.

Histopathologic examination: Examining the liver, all the groups showed cellular infiltration and congestion ranging from mild (groups III and IV), moderate (groups I and II) to severe (groups V) (Fig. 2). In addition, necrosis of the hepatocytes and diffused vacuolation of the hepatocyte were also observed in the group V (Table 2).

In the kidneys, cellular infiltration and tubular edema were the major histopathologic changes observed for all of the groups. Again, these changes were less severe in the groups III and IV (Fig. 3). Additionally, congestion, interstitial edema and focal tubular and glomerular necrosis were seen mostly among groups I, II and V when compared to groups III and IV (Table 3). Despite the long
Table 3: Histopathological changes in the kidneys seen in the various treated groups ( $n=5 /$ group)

\begin{tabular}{llllll}
\hline & \multicolumn{3}{l}{ Groups } & & \\
Lesions & I & II & III & IV & V \\
\hline Interstitial edema & 2 & 2 & 1 & - & 5 \\
Tubular edema & 3 & 2 & 2 & 1 & 5 \\
Glomerular damage & 1 & - & - & - & 3 \\
Cellular infiltration & 4 & 3 & 2 & 3 & 5 \\
Congestion & 3 & - & 1 & - & 4 \\
Focal tubular necrosis & - & - & - & - & 3 \\
\hline
\end{tabular}

Group I single subcutaneous; group II single intravenous; group III repeated subcutaneous; group IV repeated intravenous; group V negative control. The numbers in the table indicates number of dogs affected in each group

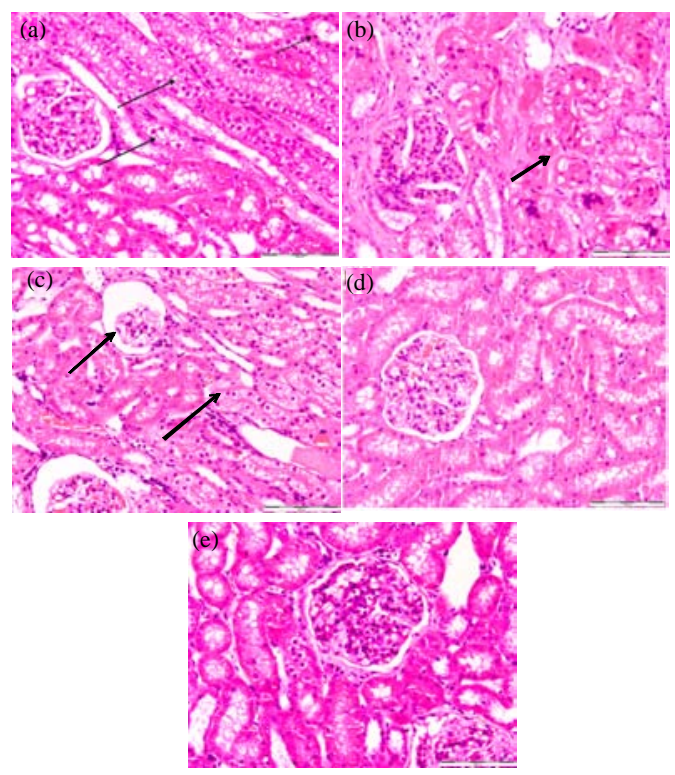

Fig. 2: a-e) High-power photomicrograph of kidney biopsy of dogs from groups V, I, II, III and IV, respectively ( $\mathrm{H}$ and $\mathrm{E} \times 100$ magnification). $\mathrm{d}$ and $\mathrm{e}$ are apparently normal kidney photomicrograph from groups III and IV, b) short arrow indicate area of hyaline cast, c) thin arrow indicate glomerular damage from group I and a) long arrow indicate tubular degeneration with vacuoles from group $\mathrm{V}$

time use of opioids in dogs, little is known on the effects of tramadol in dogs undergoing a long surgical procedure.

The primary role of liver and kidney in drug metabolism and excretion predispose these organs to toxic injury. Tramadol does increase serum ALT, AST and LDH in line with what is expected of opioids. Hence, the ability to cause hepatotoxicity and nephrotoxicity have been reported during opioids metabolism and excretion (Atici et al., 2005). In this study, ALT and AST were found to be lowered and remained within the normal range among the treatment groups (II-IV) at $4 \mathrm{~h}$ post tramadol 

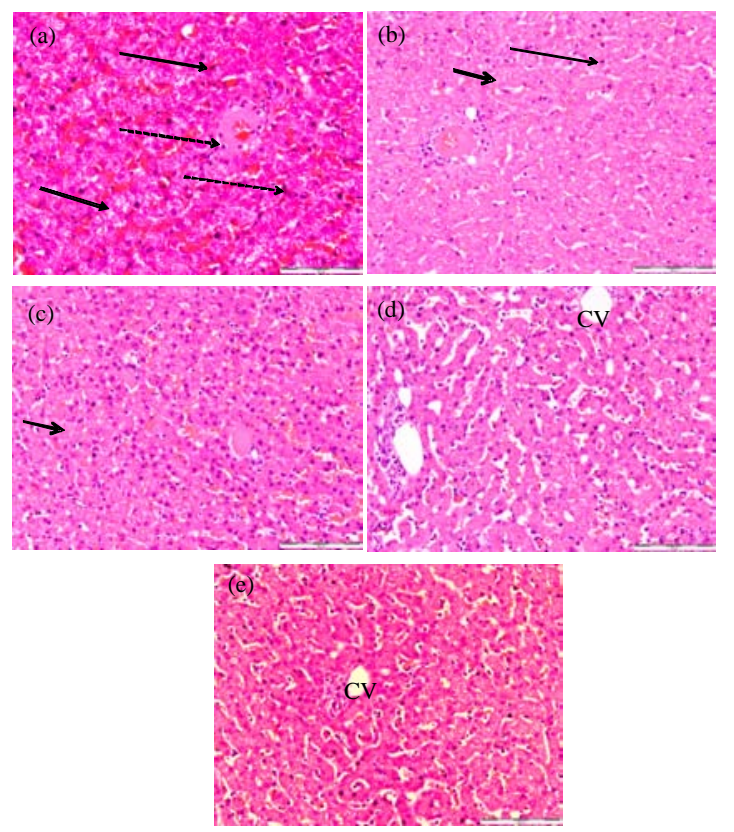

Fig. 3: a-e) High-power photomicrograph showing liver biopsy of dogs from groups V, I, II, III and IV, respectively ( $\mathrm{H}$ and $\mathrm{E}$ x100 magnification). $\mathrm{CV}$ : Central Vein, short arrow indicate area of sinusoidal congestion from groups I and II, long arrow indicate kuppfer cell, broken arrow indicate netrophils aggregation and double arrow indicate sinusoidal dilatation with a zone of mild cellular degeneration of the hepatocyte from group $\mathrm{V}$

administration compare to group V. However, an increased in AST was also observed in group I at $4 \mathrm{~h}$ post tramadol administration. The increased in ALT and AST in groups I and V were likely due to increased volume of pentobarbitone sodium (Rosenmann et al., 1972; Sato et al., 1989), rather than tramadol's effect (Atici et al., 2005; Dehkordi et al., 2010) and could be transient. Irreversible changes are unlikely to occur following therapeutic doses of opioids administration for $24 \mathrm{~h}$ in an incubated adult human hepatocytes (Gomez-Lechon et al., 1987). Significant increase in serum ALT, LDH and peroxides levels were reported among chronic heroin users (Panchenko et al., 1999). Similar observation was made in experimental mouse receiving morphine (Zhang et al., 2004) and rats placed on long-term administration of morphine-like agent, i.e., LevoAlphaacetylmethadol hydrochloride (LAAM). More so, long-term use of tramadol in rats was reported to significantly increase serum ALT level alone (Almeida et al., 2010) while increase in ALT and AST were observed in morphine groups following the same duration of administration (Atici et al., 2005). Serum BUN and creatinine levels remained unaffected throughout the experiment within the tramadol treated groups indicating that tramadol does not affect the renal function and is safe when administered repeatedly during a prolonged surgery at the recommended dose. These results verify further the notions that therapeutic doses of opioids administration are unlikely to cause irreversible changes (Gomez-Lechon et al., 1987). In addition, the histopathological changes observed in the liver and kidney biopsies were due to decreased cardiac output effect of pentobarbitone sodium, hence decreased renal blood perfusion (Wada et al., 1996). Barbiturate is reported to cause hypersensitivity reactions and biliary congestion without causing irreversible injurious effect on the hepatic function (Sato et al., 1989).

An evidence of renal damage such as tubular vacuolization, mononuclear cell infilteration, focal necrosis and haemorrahage in addition to increase serum BUN and creatinine levels in rats receiving long-term morphine administration was reported (Atici et al., 2005). Renal damage was also reported in experimental rats placed on a long-term administration of morphine-like agent LAAM (Almeida et al., 2010). On the other hand, serum BUN and creatinine levels remain unchanged among rats receiving long-term tramadol (Atici et al., 2005) and in sheep five days after tramadol administration (Dehkordi et al., 2010). However, minimal histological changes confined to the tubular cells were observed in rats receiving long-term tramadol administration (Atici et al., 2005).

No significant differences was observed throughout the groups with respect to body weight distribution, duration of anaesthesia and the duration of surgery. However, there was a significantly lowered volume of pentobarbitone sodium needed to maintain anaesthesia in groups (III and IV) receiving additional doses of tramadol. This is could be due to the fact that tramadol acted synergistically with pentobarbitone sodium and complemented its action hence lowering the amount of anaesthetic drug required to maintain surgical plane anaesthesia as reported by Seddighi et al. (2009) and Wordliczek et al. (2002) with other anaesthetic agents.

\section{CONCLUSION}

Researchers conclude that repeated administrations of tramadol at $3 \mathrm{mg} \mathrm{kg}^{-1}$, IV or SC is safe at a frequency of $2 \mathrm{~h}$ interval during a prolong surgery without causing irreversible hepatic and renal damage in healthy dogs. Similarly, repeated administrations of tramadol intraoperatively enhance analgesia thereby reducing the required dose of pentobarbitone sodium needed to maintain anaesthesia during a prolonged surgery. 


\section{REFERENCES}

Abu-Seida, A.M.A., 2012. Efficacy of diclofenac sodium, either alone or together with cefotaxime sodium, for control of postoperative pain, in dogs undergoing ovariohysterectomy. Asian J. Anim. Vet. Adv., 7: $180-186$.

Almeida, R.M., A. Escobar and S. Maguilnik, 2010. Comparison of analgesia provided by lidocaine, lidocaine-morphine or lidocaine-tramadol delivered epidurally in dogs following orchiectomy. Vet. Anaesth. Analgesia, 37: 542-549.

Atici, S., I. Cinel, L.Cinel, N. Doruk, G. Eskandari and U. Oral, 2005. Liver and kidney toxicity in chronic use of opioids: An experimental long term treatment model. J. Biosci., 30: 245-252.

Dehkordi, S.H., A.B. Sadegh, E. Abaspour, N.B. Brojeni, E. Aali and E. Sadeghi, 2010. Intravenous administration of tramadol hydrochloride in sheep: A haematological and biochemical study. Comp. Clin. Pathol. 10.1007/s00580-010-1094-2.

Gomez-Lechon, M.J., X. Ponsoda, R. Jover, R. Fabra, R. Trullenque and J.V. Castell, 1987. Hepatotoxicity of the opioids morphine, heroin, meperidine and methadone to cultured human hepatocytes. Mol. Toxicol., 1: 453-463.

Guedes, A.G.P., C.C. Natalini, E.P. Robinson, S.D.L. Alves and S.T. Oliveira, 2005. Epidural administration of tramadol as an analgesic technique in dogs submitted to stifle surgery. Int. J. Applied Res. Vet. Med., 3: $351-359$.

Kongara, K., J.P. Chambers and C.B. Johnson, 2010. Electroencephalographic responses of tramadol, parecoxib and morphine to acute noxious electrical stimulation in anaesthetised dogs. Res. Vet. Sci., 88: 127-133.

Kongara, K., P. Chambers and C.B. Johnson, 2009. Glomerular filtration rate after tramadol, parecoxib and pindolol following anaesthesia and analgesia in comparison with morphine in dogs. Vet. Anaesth. Analgesia, 36: 86-94.

Kubota, R., T. Komiyama, Y. Miwa, T. Ide, H. Toyoda, F. Asanuma and Y. Yamada, 2008. Pharmacokinetics and postoperative analgesia of epidural tramadol: A prospective, pilot study. Curr. Ther. Res., 69: 49-55.

Kukanich, B. and M.G. Papich, 2004. Pharmacokinetics of tramadol and the metabolite O-desmethyltramadol in dogs. J. Vet. Pharmacol. Ther., 27: 239-246.

Lascelles, B.D.X., 1999. Preoperative analgesia-opioids and NSAIDs. Waltham Focus, 9: 2-9.
Mastrocinque, S. and D.T. Fantoni, 2003. A comparison of preoperative tramadol and morphine for the control of early postoperative pain in canine ovariohysterectomy. Vet. Anaesth. Analgesia, 30: $220-228$.

McMillan, C.J., A. Livingston, C.R. Clark, P.M. Dowling, S.M. Taylor, T. Duke and R. Terlinden, 2008. Pharmacokinetics of intravenous tramadol in dogs. Can. J. Vet. Res., 72: 325-331.

Mercadante, S. and E. Arcuri, 2004. Opioids and renal function. J. Pain, 5: 2-19.

Panchenko, L.F., S.V. Pirozhkov, A.V. Nadezhdin, V. Baronets and N.N. Usmanova, 1999. Lipid peroxidation, peroxyl radical-scavenging system of plasma and liver and heart pathology in adolescence heroin users. Vopr. Med. Khim., 45: 501-506.

Riviere, J.E. and M.G. Papich, 2001. Potential and problems of developing transdermal patches for veterinary applications. Adv. Drug Delivery Rev., 50: $175-203$.

Rosenmann, E., T. Dishon, A. Durst and J.H. Boss, 1972. Kidney and liver damage following anaesthesia with ether and pentobarbitone. Br. J. Anaesth., 44: 465-468.

Sato, M., S. Tanaka, K. Suzuki, A. Kohama and C. Fujii, 1989. Complications associated with barbiturate therapy. Resuscitation, 17: 233-241.

Seddighi, M.R., C.M. Egger, B.W. Rohrbach, S.K. Cox and T.J. Doherty, 2009. Effects of tramadol on the minimum alveolar concentration of sevoflurane in dogs. Vet. Anaesth. Analgesia, 36: 334-340.

Tasaki, T., A. Nakamura, S. Itoh, K. Ohashi and Y. Yamamoto et al., 1998. Expression and characterization of dog CYP2D15 Using baculovirus expression system. J. Biochem., 123: 162-168.

Taylor, P., 2003. Pain management in dogs and cats: More causes and locations to contemplate. Vet. J., 165: 186-187.

Theresa, W.F., 2007. Small Animal Surgery. 3rd Edn., Mosby Elsevier, America.

Valtolina, C., J.H. Robben, J. Uilenreef, J.C. Murrell, J. Aspegren, B.C. McKusick and L.J. Hellebrekers, 2009. Clinical evaluation of the efficacy and safety of a constant rate infusion of dexmedetomidine for postoperative pain management in dogs. Vet. Anaesth. Analgesia, 36: 369-383.

Vettorato, E., A. Zonca, M. Isola, R. Villa and M. Gallo et al., 2010. Pharmacokinetics and efficacy of intravenous and extradural tramadol in dogs. Vet. J., 183: 310-315.

Wada, R.D., H. Harashima, W.F. Ebling, E.W. Osaki and D.R. Stanski, 1996. Effects of thiopental on regional blood flows in the rat. Anesthesiology, 84: 596-604. 
Wootton, R., G. Crosst, S. Wood and C.D. Westt, 1988. An analgesiometry system for use in rabbits with some preliminary data on the effects of buprenorphine and lofentanil. Lab. Anim, 22: 217-222.

Wordliczek, J., M. Banach, J. Garlicki, J. JakowickaWordliczek and J. Dobrogowski, 2002. Influence of pre- or intraoperational use of tramadol (preemptive or preventive analgesia) on tramadol requirement in the early postoperative period. Pol. J. Pharmacol., 54: 693-697.
Wunsch, L.A., B.K. Schmidt, L.A. Krugner-Higby and L.J. Smith, 2010. A comparison of the effects of hydromorphone $\mathrm{HCl}$ and a novel extended release hydromorphone on arterial blood gas values in conscious healthy dogs. Res. Vet. Sci., 88: 154-158.

Zhang, Y.T., Q.S. Zheng, J. Pan and R.L. Zheng, 2004. Oxidative damage of biomolecules in mouse liver induced by morphine and protected by antioxidants. Basic Clin. Pharmacol. Toxicol., 95: 53-58. 